\title{
Editorial 2018
}

\author{
Imre Miklós Szilágyi ${ }^{1}\left[\right.$ · Alfréd Kállay-Menyhárd ${ }^{2} \cdot$ Sophie Korda ${ }^{3}$
}

Received: 22 November 2017 / Accepted: 23 November 2017 / Published online: 1 December 2017

(c) Akadémiai Kiadó, Budapest, Hungary 2017

Dear colleagues (All contributors of Journal of Thermal Analysis and Calorimetry),

2017 was an exciting year in the life of our journal and we would like to thank all of you for your contributions as authors, reviewers, consultants as well as regional, associate, honorary and guest Editors. We are grateful to you, because your high-quality work is the guarantee for the success of our journal.

It is a great news that the impact factor for 2016 was 1.953 , which is ca. $10 \%$ increase compared to the previous year.

Several special issues were published in 2017, which include the traditional green issue where a unique chapter dedicated to Prof. Judith Simon, the honorary Editor-inChief and founder of JTAC. We continued our long-term tradition and selected papers presented at international conferences (CEEC-TAC3, AICAT2016, 11Bretszanjder) were published in JTAC in 2017.

The classification of the manuscripts submitted to our journal until October 2017 is presented in Fig. 1. We are very happy that the number of manuscript submitted in 2017 is expected to be around 1800 pieces, which is similar to 2016.

In 2017, the journal established a new international conference series on thermal analysis and calorimetry, the Journal of Thermal Analysis and Calorimetry Conference (JTACC). During the almost five decades of publishing, a global community formed around JTAC, and we wished to

Imre Miklós Szilágyi

imre.szilagyi@mail.bme.hu

1 Department of Inorganic and Analytical Chemistry, Budapest University of Technology and Economics, Szt. Gellért tér 4, Budapest 1111, Hungary

2 Department of Physical Chemistry and Materials Science, Budapest University of Technology and Economics, P.O. Box 92, Budapest 1521, Hungary

3 Akadémiai Kiadó, Prielle K. u. 21-35, Budapest 1117, Hungary create an opportunity, where our editorial board, authors, reviewers, supporters, and all those who were interested in this wonderful field, could meet personally. As a follow-up of the meeting, selected presentations will be published in JTAC.

We thank everybody very much for participating at the 1st Journal of Thermal Analysis and Calorimetry Conference, which was held together with the 6th V4 (Joint Czech-Hungarian-Polish-Slovakian) Thermoanalytical Conference, in Budapest on June 6-9, 2017.

We had a wonderful time together at the JTACC + V4 conference where 126 plenary invited and contributed lectures and 173 posters were presented. Ca. 300 participants attended the conference from close to 50 countries from all over the world.

At the conference, three awards were handed over. Our traditional JTAC Best reviewer Award was given to Prof. Katalin Mészáros-Szécsényi (University of Novi Sad, Serbia). In addition, we established two new grants, and thus the JTAC Scientific Excellence Award was given to Prof. Jean Rouquerol (emeritus research director of the Centre Nacional de la Recherche Scientifique (CNRS), France), while the JTAC Young Scientist Award to Dr. Omid Mahian (King Mongkut's University of Technology Thonburi, Thailand).

We are pleased to announce the 2nd JTACC conference, which will be held in 2019. It will be a very special meeting as we will celebrate the 50th anniversary of the Journal of Thermal Analysis and Calorimetry. Due to this, the conference will be held in Hungary, where JTAC was founded and where also the first commercial simultaneous TG/DTA device of the world, the famous Derivatograph was developed.

In 2018, we would like to increase further the quality of our journal; hence, we have decided on several changes. The most important is that we completely renew our editorial board, which has been the same for nearly a decade. Up to now, we had associate, regional and honorary editors. From 2018, our editorial board will consist of associate and honorary editors with well-defined roles (e.g., decisive review reports, research and review paper 


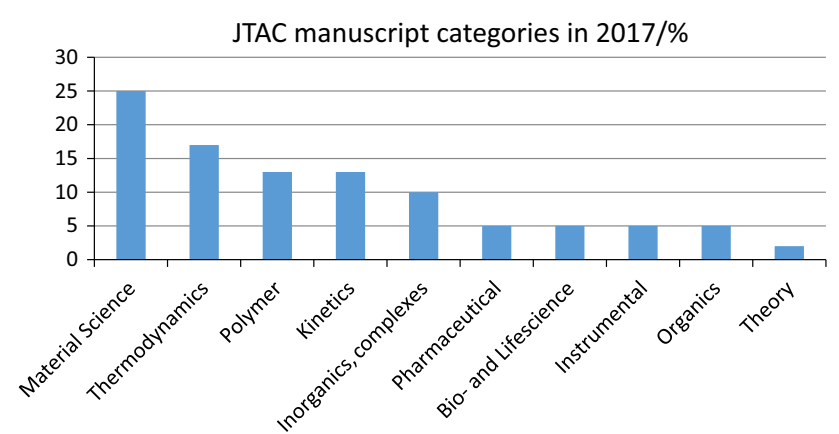

Fig. 1 The classification of the manuscripts submitted to our journal in 2017

submission, special influence on shaping both JTAC journal quality and trends in thermal analysis and calorimetry, popularizing JTAC). We will reduce the various deadlines in the editorial process (e.g., reviewer invitation deadline, review report deadline) to please our authors even more by accelerating editorial speed. From 2018, we will publish only research articles and review papers, while we will not consider short communications.

As a closing remark, we would like to wish all our contributors a very happy and successful new year for 2018.

$$
\begin{array}{r}
\text { Giling } 2 \\
\text { Imre Miklós Szilágyi } \\
\text { Editor-in-Chief }
\end{array}
$$

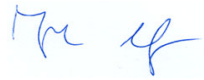

Alfréd Kállay-Menyhárd

Deputy Editor-in-Chief

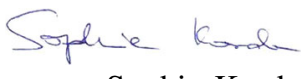

Sophie Korda

Head of Editorial Office 


\section{Acknowledgements}

The editors of the journal are thankful to the regional editors and members of the associate editors for their work throughout the past year.

We would like to express our special thanks to those referees listed below for their hard work, for the time and expertise they invested to improve the scientific level of the manuscripts. Their work is invaluable in terms of scientific advancement and in providing a constant dynamic forum where all scientists, from all over the world can find a professional team helping, educating and inspiring.

Abate, L., Italy

Abbas Abadi, M. S., Iran

Abbasian Arani, A. A., Iran

Abd El-halim, H. F., Egypt

Abdel-Kader, N. S., Egypt

Abdul, J., India

Abo Zeid, E. F., Egypt

Abou-Melha, K., Egypt

Acar, I., Turkey

Achilias, D. S., Greece

Adil, A., Morocco

Adnadjevic, B., Serbia

Afrand, M., Iran

Agostini, D. L. S., Brazil

Ahmadi, M., Iran

Ahmed, J., Kuwait

Akbari, M., Iran

Akhtar, S., Bangladesh

Akinlami, J., UK

Akpinar, E., Turkey

Aksu Canbay, C., Turkey

Al-Harthi, M. A., Saudi Arabia

Al-Kouz, W., Jordan

Al-Sarkhi, A. S., Iran

Albis, A., Colombia

Albu, P. A., Romania

Alex, R., India

Alghool Alghool, S., Egypt

Alkan, C., Turkey

Alonso, V., Spain

Alshehri, S. M., Saudi Arabia

Alves Rocha, E. P., Brazil

Ambrozini, B., Brazil

Amer, A., Egypt

Amim Jr., J., Brazil

Amin, S. K., Egypt

Amiri, A., Malaysia

An, W., China

Anacona, J. R., Venezuela

Anand, S., India

Andrade Nogueira, F. H., Brazil

Anghel, E. M., Romania

Antonovic, V., Lithuania
Ao, W., China

Araujo, A. S., Brazil

Araujo, R. C., Brazil

Araújo Sales, M. J., Brazil

Arico, E. M., Brazil

Arora, C., India

Arslan, H., Turkey

Asabina, E. A., Russian Federation

Artiaga, R., Spain

Ashraf, W., USA

Ataíde, C. H., Brazil

Atakol, M., Turkey

Atanasova, L. G., Bulgaria

Atiqullah, M., Saudi Arabia

Atkinson, I., Romania

Auroux, A., France

Avar, B., Turkey

Avdeev, G., Bulgaria

Avramov, I., Bulgaria

Ayachit, N., India

Aydin, O., Turkey

Aydinyan, S., Armenia

Aydogdu, Y., Turkey

Ayodele, B. V., Malaysia

Azmi, S., Canada

Bachaga, T., Tunisia

Badea, E., Italy

Badea, M. E., Romania

Baghaie, S., Iran

Bahiraei, M., Iran

Bai, Z., China

Bailleul, J.-L., France

Bal, S., Turkey

Bala Sharma, K., India

Balc1, Y., Turkey

Balkose, D., Turkey

Baloda, S., India

Baltakys, K., Lithuania

Bamzai, K. K., India

Bankauskaite, A., Lithuania

Bannach, G., Brazil

Barbosa, R., Brazil

Barreto Gomes, A. P., Brazil 
Barta Holló, B., Serbia

Bartyzel, A., Poland

Bauer, J., Poland

Bedor, D., Brazil

Belhouchet, H., Algeria

Bellich, B., Italy

Bellitto, V., USA

Benziane, M., Algeria

Berbenni, V., Italy

Bhagavath, P., India

Bharadwaj, S., India

Bhattacharya, S., USA

Bih, L., France

Bikiaris, D., Greece

Biliškov, N., Croatia

Billingham, N., UK

Bilyeu, B., USA

Blaine, R., USA

Blanco, I., Italy

Blanco Varela, M. T., Spain

Blazejowski, J., Poland

Blanco Varela, M. T., Spain

Blonska-Tabero, A., Poland

Bobrowicz, J., Poland

Boerner, E., Poland

Bohn, M. A., Germany

Bohre, A., India

Bollikolla, H. B., India

Bonk, A., Germany

Borek, W., Poland

Borsa, J., Hungary

Boyard, N., France

Bozzelli, J. W., USA

Brahadeeswaran, S., India

Braissant, O., Switzerland

Braz Fernandes, F., Portugal

Bright, C., India

Brocardo Machado, L. D., Brazil

Brodarac, Z. Z., Croatia

Brozek, J., Czech Republic

Bruni, G., Italy

Bryś, A., Poland

Budrugeac, P., Romania

Bulanek, R., Czech Republic

Burnham, A. K., USA

Buzutti Siqueira, A., Brazil

Cabaleiro, D., Spain

Cai, X.-f., China

Cai, Y., China

Caires Jr., F., Brazil

Calado, V. M. A., Brazil

Camacho Rodrigues, A., Brazil

Cao, Y., USA
Cardinale, A. M., Italy

Cardoso Rodrigues Santos, K. S., Brazil

Carvalho, C. T., Brazil

Cavalheiro, E., Brazil

Cavallaro, G., Italy

Cavallo, D., Italy

Cecilia, J. A., Spain

Celebi Efe, G. F., Turkey

Cerc Korošec, R., Slovenia

Cerdeirina, C., Spain

Cerny, R., Czech Republic

Cervantes-Uc, J. M., Mexico

Chadha, R., India

Chang, J., China

Chang, J.-H., China

Charmas, B., Poland

Che, D., China

Chellappa, T., Brazil

Chen, B., China

Chen, C., China

Chen, C., USA

Chen, H. X., China

Chen, L., China

Chen, M., China

Chen, S., Australia

Chen, T., China

Chen, W., China

Chen, X., China

Chen, X., USA

Cheng, C. K., Malaysia

Cheng, F., China

Cheng, H., China

Cheng, J., China

Cheng, X., China

Cheng, Y., China

Chieruzzi, M., Italy

Chmielarz, L., Poland

Chojnacka, I., Poland

Cholewka, A., Poland

Chow, W. S., Malaysia

Chowdhury, A., India

Chrissafis, K., Greece

Christelle, G., France

Chromčíková, M., Slovakia

Chu, M., China

Cibulkova, Z., Slovakia

Ciobanu, C., Romania

Circu, V., Romania

Comyn, J., UK

Conceicaio, M. M., Brazil

Conesa, J. A., Spain

Corbellini, V. A., Brazil

Crespi, M. S., Brazil 
Criado, J. M., Spain

Crnkovic, P. M., Brazil

Cucos, A., Romania

Cunha Filho, M. S. S., Brazil

Czakiert, T., Poland

Czylkowska, A., Poland

Cser, F., Australia

da Guarda Souza, M. O., Brazil

da Silva, E. A., Brazil

da Silva, R. A. G., Brazil

da Silva Jr., A. A., Brazil

Dağdelen, F., Turkey

Dalla Rosa, M., Italy

Dantas Medeiros, A. C., Brazil

Dash, S., India

Datta, J., Poland

Davalos, J. Z., Spain

De Carvalho Anjos, V., Brazil

de Godois Baroni, É., Brazil

de Klerk, W., The Netherlands

De Melo Azevedo, E., Brazil

de Moura Torquato, L. D., Brazil

De Oliveira Tiera, V. A., Brazil

Deb, N., India

Dékány, I., Hungary

Demetzos, C., Greece

Demir, H., Turkey

Denck Colman, T. A., Brazil

Deng, T., China

Derylo-Marczewska, A., Poland

Di, Y.-Y., China

Di Martino, P., Italy

Dias Santos, A. G., Brazil

Diwu, J., China

Djangang, C. N., Cameroon

Dmitrii, P., Russian Federation

Doca, N., Romania

Dogan, M., Turkey

Dohnalová, Ž., Czech Republic

Dóka, O., Hungary

Domján, A., Hungary

Dong, C., China

Dong, G., China

Dong, Y., China

Dos Santos Muccillo, E. N., Brazil

dos Santos Politi, J. R., Brzail

Dou, Q., China

Drebushchak, V. A., Russian Federation

Drienovsky, M., Slovakia

Drouet, C., France

Druzhinina, A., Russian Federation

Drzazga, Z., Poland

Držková, M., Czech Republic
Du, R., China

Dubaj, T., Slovakia

Dubaniewicz, T. H., USA

Dubey, R., USA

Duce, C., Italy

Duh, Y.-S., Taiwan

Dumitru, F., Romania

Dumitru, R., Romania

Durmus, H., Turkey

Durrani, S. K., Pakistan

Durusoy, T., Turkey

Dweck, J., Brazil

Dziejowski, J. E., Poland

Ebadi-Dehaghani, H., Iran

Edwin, M., India

El-Bindary, A. A.-A., Egypt

El-Gamal, S. M. A., Egypt

El-Medani, S., Egypt

El-Nahrawy, A. M., Egypt

El-Oyoun Hassan, M. A., Saudi Arabia

El-Sayed Ain, M., Egypt

El-Sayed Hekal, E., Egypt

El-Shahat, M. F., Egypt

El-Sonbati, A., Egypt

Ellahi, R., USA

Engelen, B., Germany

Erdogan, B. C., Turkey

Eren, T., Turkey

Ermolina, I., UK

Ershad-Langroudi, A., Iran

Esfandeh, S., Iran

Esfe, M. H., Iran

Eslami, A., Iran

Esperanza, M., Spain

Estellé, P., France

Evrendilek, G. A., Turkey

Ezz Eldin, F. M., Egypt

Fabia, J., Poland

Falconer, R. J., UK

Fang, G. Y., China

Fang, H., China

Fang, Z., China

Farahany, S., Malaysia

Farkas, A., Hungary

Fatehy El-Shahat, M., Egypt

Favergeon, L., France

Feng, J., China

Feng-Qi, Z., China

Fengqi, Z., China

Ferenc, W., Poland

Ferencz, A., Hungary

Fernandez Berridi, M. J., Spain

Ferry, L., France 
Fertonani, F. L., Brazil

Fiamingo, A., Brazil

Fierascu, I., Romania

Filip, J., Czech Republic

Fima, P., Poland

Findorák, R., Slovakia

Firouzi, M., Iran

Fitzner, K., Poland

Flores, H., Mexico

Focke, W. W., South Africa

Fokaides, P. A., Cyprus

Fonseca Costa, M. J., Brazil

Frache, A., Italy

França Holanda, J. N., Brazil

Frencisc, P., Romania

Frost, R. L., Australia

Fu, X. L., China

Gac, W., Poland

Gahleitner, M., Austria

Galán, J. J., Spain

Ganapathi, A. S., Singapore

Gao, C., China

Gao, L., China

Gao, M., China

Gao, S.-L., China

Garbett, N., USA

Garcia-Sanchez, E., Mexico

Gathania, A., India

Gaune-Escard, M., France

Gaur, M. S., India

Gavrichev, K. S., Russian Federation

Gawdzik, B., Poland

Genc, Z. K., Turkey

Ghalsasi, P., India

Ghislain, D., France

Ghosh, A., India

Ghosh, K. S., India

Ghyngazov, S., Russian Federation

Gialanella, S., Italy

Giancola, C., Italy

Gingu, O., Romania

Golestaneh, A. F., Canada

Gołofit, T., Poland

Gómez-de la Cruz, F. J., Spain

Gómez-Serrano, V., Spain

Goncalves, C., Brazil

Gonçalves Mothé, C., Brazil

Goncharuk, O., Ukraine

Gong, P., China

Górniak, A., Poland

Gorodylova, N., Czech Republic

Górska, A., Poland

Govindarajan, S., India
Gozin, M., Israel

Grabowska, B., Poland

Graczyk, T., Poland

Greco, A., Italy

Grigiante, M., Italy

Grochowicz, M., Poland

Gróf, Gy., Hungary

Guan, W., China

Guettari, M., Tunisia

Guimarães, D., Brazil

Guo, F., China

Guo, J., China

Guo, W., China

Gupta, J. P., India

Gyarmati, B., Hungary

Győryová, K., Slovakia

Habibi, M. H., Iran

Hacaloglu, J., Turkey

Hajmohammad, M. H., Iran

Hamed, E., Egypt

Hameed, A., Pakistan

Han, C., China

Han, X., China

Han, Z., China

Hao, J., China

Haseli, Y., USA

Hashem, F. S., Egypt

Hassanien, A. S., Egypt

Hatakeyama, T., Japan

Hatanpää, T., Finland

Haudin,J.-M., France

Hay, J. N., UK

He, Y., China

Heikal, M., Saudi Arabia

Hemmat Esfe, M., Iran

Heris, S. Z., Iran

Herrera-Franco, P. J., Mexico

Hnilicka, F., Czech Republic

Hofmeister, A., USA

Holakooei, P., Iran

Holanda, J. N. F., Brazil

Holubová, J., Czech Republic

Honcova, P., Czech Republic

Hongbo, L., China

Hosny, N., Egypt

Hosseini, S. G., Iran

Howell, B. A., USA

Hsu, C.-K., Taiwan

$\mathrm{Hu}, \mathrm{R} .$, China

$\mathrm{Hu}$, S., China

$\mathrm{Hu}, \mathrm{W}$. , China

$\mathrm{Hu}, \mathrm{Y}$. , China

Hua, W., China 
Huang, L., China

Huang, S., China

Huang, W., USA

Huang, X., China

Huang, Z., China

Hui, R., China

Hussaini Syed, S. S., India

Hutchinson, J. M., Spain

Iijie, I., China

Ikeda, H., Japan

Ionashiro, E. Y., Brazil

Ionashiro, M., Brazil

Ionescu, N. I., Romania

Ipek, M., Turkey

Ipser, H., Austria

Iqbal, M. S., Pakistan

Iqbal, S. S., Pakistan

Irassar, E. F., Argentina

Ismail, K., India

Iwama, S., Japan

Izato, Y.-i., Japan

Jablonski, M., Poland

Jaiswar, G., India

Janković, B., Serbia

Jha, V., USA

Ji, J., China

Jia, H., China

Jia, Y., UK

Jia, Y.-Z., China

Jiang, J., USA

Jiang, X., China

Jiang, Y., China

Jiao, T., China

Jin, B., China

Jitianu, A., USA

Jones Silva, M., Brazil

Jovanovic, J. D., Serbia

Jozwiak, M., Poland

Judovits, L., USA

Jun, Y., China

Junca, E., Brazil

Justin Raj, C., India

Kabir, K. B., Bangladesh

Kaczmarek, H., Poland

Kalaee, M., Iran

Kaletunc, G., USA

Kalia, S. B., India

Kaljuvee, T., Estonia

Kan, C., China

Kandasamy, J., France

Kandeel, M., Japan

Kani, E. N., Iran

Kannan, M., India
Kappenstein, C., France

Kaptay, Gy., Hungary

Karami, K., Iran

Karasu, S., Turkey

Karger-Kocsis, J., Hungary

Karolewicz, B., Poland

Károly, Z., Hungary

Katakam, M., India

Kataki, R., India

Kawai, T., Japan

Kaygili, O., Turkey

Kazi, S. N., Malaysia

Kelnar, I., Czech Republic

Kenisarin, M., UK

Kennedy, A., USA

Kerch, G., Latvia

Kerekes, Zs., Hungary

Kéri, O., Hungary

Keshavarz, M., Iran

Khalameida, S., Ukraine

Khan, I., Oman

Kheradmand, M., Portuga

Khimeche, K., Algerial

Kim, S., Korea

Kimura, A., Japan

Kimura, T., Japan

Kirichenko, O., Russian Federation

Kizilca, M., Turkey

Klapotke, T. M., Germany

Klemczak, B. A., Poland

Knyazev, A. V., Russian Federation

Kobelnik, M., Brazil

Koçak, S. B., Turkey

Koga, N., Japan

Kohoutková, M., Czech Republic

Kolaitis, D. I., Greece

Kolekar, S. S., India

Koleva, V., Bulgaria

Koltsov, I.E., Poland

Kong, Q., China

Kong, W., China

Kong, X., China

Kooli, F., Saudi Arabia

Kopecskó, K., Hungary

Kótai, L., Hungary

Kotkata, M. F., Egypt

Kozlov, A., Russian Federation

Kozowłska, M., Poland

Kök, M., Turkey

Kök, M. V., Turkey

Krajewski, W. K., Poland

Kristóf, J., Hungary

Król, D. J., Poland 
Krongauz, V. V., USA

Krumova, S., Bulgaria

Krunks, M., Estonia

Krupinski, M., Poland

Kruszynski, R., Poland

Ksepko, E., Poland

Kucerik, J., Germany

Kumar, A., India

Kumar, V., India

Kumar Maheswaram, M. P., USA

Kumar Sen, A., India

Kumar Swamy, N., India

Kun, D., Hungary

Künzel, M., Czech Republic

Kurajica, S., Croatia

Kurt, H., Turkey

Kuśtrowski, P., Poland

Labus, M., Poland

Lacerda, L., Brazil

Lafuente, C., Spain

Lager, D., Austria

Lal, S., India

Lalia-Kantouri, M., Greece

Lan, X., China

Lanceros-Mendez, S., Portugal

Larochette, P. A., Argentina

Lavasani, A., Iran

Lazzara, G., Italy

Lazzarotto, M., Brazil

Leandro, A., Brazil

Ledeti, A., Romania

Ledeti, I. V., Romania

Lee, K.-S., Korea

Lee, P., Canada

Legrand, V., France

Leveneur, S., France

Li, D., China

Li, F., China

Li, H., China

Li, J., China

Li, K. Y., Finland

Li, L., China

Li, N., China

Li, P., China

Li, Q., China

Li, Q.-G., China

Li, S., China

Li, Y. F., China

Liang, T., China

Liao, S., China

Liao, Y. G., China

Liaw, H.-J., Taiwan

Lim, A. R., Korea
Lima Barros de Araujo, G., Brazil

Lima Gomes, E. C., Brazil

Lin, B., China

Lin, C., China

Lin, C.-P., Taiwan

Lin, Z., China

Linert, W., Austria

Lipkowski, J. S., Poland

Lisa, G., Romania

Liska, M., Slovakia

Lisnyak, V. V., Ukraine

Litaiem, H., Tunisia

Liu, C., China

Liu, C., Spain

Liu, F., China

Liu, F. R., China

Liu, J.-Z., China

Liu, K.-C., Taiwan

Liu, L., China

Liu, L.-L., China

Liu, M., China

Liu, N., China

Liu, P., China

Liu, P.-J., China

Liu, Q., China

Liu, W., China

Liu, X., China

Liu, Y., China

Liu, Z., China

Lochab, B., India

Logvinenko, V. A., Russian Federation

Lomonaco, D., Brzail

Lörinczy, D., Hungary

Lu, G., China

Lu, L., Australia

Lu, L., China

Lu, M., China

Lu, P., China

Lu, Q., China

Lu, S., China

Lu, X., China

Lu, Y., China

Lublóy, É. E., Hungary

Lura, P., Switzerland

Lv, X., China

Lv, X.-C., China

Lyon, R. E., USA

Lysenko, E., Russian Federation

Łszczek, R., Poland

Ma, H., China

Ma, S., China

Ma, Y., China

Macedo, Z. S., Brazil 
Machowska, A., Poland

Madarász, J., Hungary

Madej, D., Poland

Madkour, M., Kuwait

Magdalena, A. G., Brazil

Magdziarz, A., Poland

Mahadevan, S., India

Maheswaram, M. P. K., USA

Maheswaran, S., India

Mahian, O., Thailand

Mahmoud, W. H., Egypt

Mai, K., China

Majchrzak-Kuceba, I., Poland

Majoni, S., Zimbabwe

Małecki, S., Poland

Malek, J., Czech Republic

Malghe, Y., India

Małgorzata, W., Poland

Malow, M., Germany

Malucelli, G., Italy

Malvandi, A., Iran

Mansour, A. M., Egypt

Mansour, S. A., Egypt

Mantheni, D., USA

Mao, Z., China

Maria, T., Portugal

Markin, A. V., Russian Federation

Marosi, Gy., Hungary

Marossy, K., Hungary

Martínez-Hernández, A. L., Mexico

Martinka, J., Slovakia

Martins, F. T., Brazil

Mary Deborrah, S. P., India

Maryandyshev, P., Russian Federation

Mashaei, P. R., Iran

Maskow, T., Germany

Masoud, M. S., Egypt

Maswadeh, H., Saudi Arabia

Materazzi, S., Italy

Matskevich, N., Russian Federation

Matsuo, T., Japan

Matulis, D., Lithuania

Maurya, R. C., India

Meenakshisundaram, S., India

Meeyoo, V., Thailand

Meghea, A., Romania

Mehta, N., India

Meloni, D., Italy

Meltzer, V., Romania

Mendes, L. C., Brazil

Mendes, R., Brazil

Menezes, P., Brazil

Meng, Z., China
Mészáros Szécsényi, K., Serbia

Mi, J., China

Mianowski, A., Poland

Michnik, A., Poland

Minea, A. A., Romania

Ming, Y., USA

Mintz, E. A., USA

Miyazaki, H., Japan

Mocioiu, O. C., Romania

Moczo, J., Hungary

Mohamad, A. Z., Malaysia

Mohammad, S., Iran

Mohamed, G. G., Egypt

Mohamed, M. A., Egypt

Mohanan, P. V., India

Mokhtar, M., Egypt

Monajjemzadeh, F., Iran

Mondal, P., India

Montazeri, A., Iran

Montedo, O. R. K., Brazil

Monteiro, E., Portugal

Montoya Urbina, M., Brazil

Morancho, J. M., Spain

Moreira Lira, A. A., Brazil

Moreno-Piraján, J. C., Colombia

Mori, T., Japan

Moskal, G. J., Poland

Mousa, M., Egypt

Mousavian, R. T., Iran

Mousaviazar, A., Iran

Mucsi, G., Hungary

Muller, A. J., Venezuela

Muraleedharan, K., India

Muresan, L. E., Romania

Murshed, S., Portugal

Mustata, F., Romania

Musuc, A. M., Romania

Nabi Aly, K. A., Egypt

Nagarajan, K., India

Nagy, B., Hungary

Nagygyörgy, V., Hungary

Nagyné Kovács, T., Hungary

Narula, K., India

Nascimento, T. G., Brazil

Nasiruddin Khan, M., Pakistan

Naskar, M. K., India

Nassar, M., Egypt

Natali, M., Italy

Nayeem, S. M., India

Necefoğlu, H., Turkey

Németh, P., Hungary

Nespoli, A., Italy

Neugebauer, F., Germany 
Neves Jr., A., Brazil

Neves de Lima, Á. A., Brazil

$\mathrm{Ni}, \mathrm{X}$., China

Niccoli, M., Italy

Nicolopoulos, S., Belgium

Nidhi, A. V., India

Nie, S., China

Nieto, J., Spain

Ninago, M., Argentina

Ninov, J., Bulgaria

Nocun-Wczelik, W., Poland

Noisong, P., Thailand

Norquist, A., USA

Nour El-Dien, F. A., Egypt

Nowicki, P., Poland

Nunes, L., Brazil

Nunes, R. S., Brazil

Odlyha, M., UK

Okumuş, M., Turkey

Olar, R., Romania

Olewnik-Kruszkowska, E., Poland

Oliveira, M. A., Brazil

Oliveira da Guarda Souza, M., Brazil

Oliveira Macedo, R., Brazil

Omrani, A., Iran

Orozco-Guareno, E., Mexico

Osajima, J., Brazil

Ostrovskii, V. E., Russian Federation

Otero, M., Spain

Overdeep, K. R., USA

Oxley, J., USA

Ozao, R., Japan

Önal, M., Turkey

Ortiz, E., Colombia

Otero, M., Spain

Oudadesse, H., France

Pabuccuoglu, S., Turkey

Pacewska, B., Poland

Palmero, P., Italy

Palou, M., Slovakia

Pan, W.-P., USA

Pandey, M., India

Paniagua, S., Spain

Pantani, R., Italy

Pantoya, M., USA

Panwar, N. L., India

Paolini, A. E., Italy

Papadaki, M., Greece

Papageorgiou, G. Z., Greece

Paramanandam, T., India

Parida, S. C., India

Parisi, F., Italy

Parlak, T. T., Turkey
Parsania, P. H., India

Partyka, J., Poland

Patel, M. N., India

Paul, B., India

Pekar, M., Czech Republic

Pereira da Silva, M. L., Brazil

Pereira Gonçalves, J., Brazil

Pérez, J. P., Spain

Perez Gonzalez, E., Brazil

Perez-Casas, S., Mexico

Perez-Lopez, O. W., Brazil

Pérez-Maqueda, L., Spain

Pergal, M., Serbia

Perlovich, G., Russian Federation

Petkova, V., Bulgaria

Petreanu, I., Romania

Petrič, M., Slovenia

Pielichowski, K., Poland

Piloto-Rodriguez, R., Cuba

Pimerzin, A., Russian Federation

Pintye-Hódi, K., Hungary

Pivarciova, E., Slovakia

Plano, D., Spain

Plevová, E., Czech Republic

Podbielska, H., Poland

Podgórski, M. A., Poland

Podkościelna, B., Poland

Poklar Ulrih, N., Slovenia

Polguj, M., Poland

Pop, N., Romania

Popa, A., Romania

Popa, V. T., Romania

Popescu, A. M., Romania

Popescu, C., Germany

Porcedda, S., Italy

Pouretedal, H. R., Iran

Pourmortazavi, S. M., Iran

Pretrel, H., France

Princi, E., Italy

Prisciandaro, M., Italy

Prokof'ev, V. Y., Russian Federation

Pruchnik, H., Poland

Punia, R., India

Puziy, A., Ukraine

Pyda, M., USA

Qian, H., China

Qian, L., China

Qian, X., China

Qiang, W., China

Qiang, Z., USA

Qiao, Z., China

Qin, J., China

Qin, S., China 
Qiu, X., China

Qiu, Z., China

Qu, H., China

Rajagopalan, S., India

Rajeev, R., India

Raka, L., Macedonia

Ramadan, M. M., Egypt

Ramadan, R., Egypt

Rambaldi, E., Italy

Ramesan, M. T., India

Rapacz-Kmita, A., Poland

Rashidi, M. M., China

Rashidi, M. M., UK

Rattanasak, U., Thailand

Ravinder, D., India

Ravindra Prasad, M. R., India

Redkin, A., Russian Federation

Refat, M. S., Saudi Arabia

Regdon Jr., G., Hungary

Relkin, P., France

Ren, J.-G., USA

Ren, Q., China

Ren, X., China

Ribeiro, C. A., Brazil

Ribeiro da Silva, M. D. M. C., Portugal

Rietveld, I. B., France

Righi, L., Italy

Rimez, B., Belgium

Risoluti, R., Italy

Ristić, I. S., Serbia

Rivera, J., Mexico

Rivers, G. S. M., Canada

Rogulska, M., Poland

Rostamian, H., Iran

Rosu, D., Romania

Rotaru, A., Romania

Rotaru, P., Romania

Rouquerol, J., France

Roy, M., India

Rudolfovna Usacheva, T., Russian Federation

Rybinski, P., Poland

Rycerz, L., Poland

Rychly, J., Slovakia

Ryu, H.-J., Korea

Rzepa, G., Poland

Saad, G., Egypt

Sabbar, A., Morocco

Sabeur, H., Tunisia

Sadaka, S., USA

Sade, M., Argentina

Sadeek, S. A., Egypt

Sádovská, G., Czech Republic

Saengsuwan, S., Thailand
Safaei, M., Viet Nam

Sagdinc, S., Turkey

Saha, A., USA

Saikrasun, S., Thailand

Sakizci, M., Turkey

Sakthipandi, K., India

Salama, N., Egypt

Salunke, S. A., India

Salvado, M., Portugal

Samouillan, V., France

Sanchez-Jimenez, P., Spain

Sanchez-Silva, L., Spain

Sánta, R., Serbia

Santulli, C., Italy

Saraswat, S., India

Sarawat, V. K., India

Saroja, S., India

Sasaki, T., Japan

Sasca, V. Z., Romania

Sergeyevna Sashina, E., Russian Federation

Satheeshkumar, N., India

Sato, Y., Japan

Sattari, M., South Africa

Savija, B., The Netherlands

Savu, D. I., Romania

Schartel, B., Germany

Schawe, J., Switzerland

Schindler, A., Germany

Schiraldi, A., Italy

Schmelzer, J. W. P., Germany

Schmit, H. R., Germany

Schnitzler, E., Brazil

Sebastião, R. C. O., Brazil

Sebestyén, Z., Hungary

Sedmidubsky, D., Czech Republic

Segall, A. I., Argentina

Segatto Silva, M. A., Brazil

Seguela, R., France

Sekhar, J., India

Sencadas, V., Australia

Seo, Y., Korea

Servant, C., France

Šesták, J., Czech Republic

Setua, D. K., India

Shabestari, S., Iran

Shah, P. K., USA

Shaheer Akhtar, M., Korea

Shalaev, E. Y., USA

Shanahan, N., USA

Shanbedi, M., Iran

Sharma, R., Malaysia

Sharma, V. K., India

Shen, W., India 
Shen, Y., China

Sheremet, M., Russian Federation

Sherif, Y., Egypt

Shi, L., Singapore

Shi, Q., China

Shi, X., USA

Shiota, K., Japan

Shoushtari, A. M., Iran

Shu, C.-M., Taiwan

Shu, Q., China

Shuai, Q., China

Shuhua, L., China

Shuttleworth, P., Spain

Siauciunas, R., Lithuania

Sieradzki, A., Poland

Sieroń-Stoltny, K., Poland

Sikorska, E., Poland

Siligardi, C., Italy

Silva Caldeira, V. P., Brazil

Silva Leonardo, R., Brazil

Šimon, P., Slovakia

Sina, H., Sweden

Sinditskii, V. P., Russian Federation

Singh, A. V., India

Singh, C. P., India

Singh, G., India

Singh, G., USA

Singh, K., India

Singh, O. K., India

Singh, N. B., China

Singh, N. B., India

Singh, P. K., India

Singh Dahiya, M., India

Singh Gaur, M., India

Sinha, Y. K., India

Sirotkin, V., Russian Federation

Siudyga, T., Poland

Skirtach, A., Belgium

Skórczewska, K., Poland

Skwarek, E., Poland

Smetana, B., Czech Republic

Soares Cunha Filho, M. S., Brazil

Soares-Sobrinho, J. L., Brazil

Sobczak, P., Poland

Sohár, G., Hungary

Sokolowski, J., Canada

Soliman, M. H., Egypt

Song, X., China

Soria-Verdugo, A., Spain

Sousa Epaminondas, P., Brazil

Souza, A. L., Brazil

Souza Araújo, A. A., Brazil

Souza Rastelli, A. N., Brazil
Sovizi, M. R., Iran

Spasojevic-de Biré, A., France

Spassov, T., Bulgaria

Sreehari Sastry, S., India

Srinivasan, B. R., India

Srinivasan, K., India

Srinivasulu, M., India

Stambolova, I., Bulgaria

Stankovic, S. B., Serbia

Starykh, R., Russian Federation

Staszczuk, P., Poland

Steiner Petrovi, D., Slovenia

Stepanov, I., Latvia

Stoia, M. E., Romania

Stoilova, D., Bulgaria

Stonys, R., Lithuania

Strydom, C., Algeria

Suceska, M., Croatia

Sudarsan, V., India

Suksaeree, J., Thailand

Sulcova, P., Czech Republic

Sulowska, J., Poland

Sun, B., China

Sun, F.,USA

Sun, J., China

Sun, L., China

Sun, L., USA

Suñol, J. J., Spain

Suplicz, A., Hungary

Sutcu, H., Turkey

Svoboda, R., Czech Republic

Swamy, N. K., India

Świderski, G., Poland

Syal, V. K., India

Szabó-Révész, P., Hungary

Szczes, A., Poland

Szczygieł, I., Poland

Szeluga, U., Poland

Szépvölgyi, J., Hungary

Szumera, M., Poland

Talawar, M. B., India

Tamizi, E., Iran

Tan, Z., China

Tan, Z.-C., China

Tanaka, R., Japan

Tancredi, N., Uruguay

Tang, K., China

Tangeda, S. J., India

Tao, Q., China

Taraba, B., Czech Republic

Tarantili, P. A., Greece

Tashima, M. M., Brazil

Telles, V. B., Brazil 
Temel, H., Turkey

Terekhova, I. V., Russian Federation

Tertykh, V., Ukraine

Thangavel, V. C., India

Thomas, P., Australia

Thomas, P. C., India

Thomas, S., USA

Timelli, G., Italy

Tita, B., Romania

Tiverios, V., Greece

Tiwari, G. P., India

Toghraie, D., Iran

Tomaszewicz, M., Poland

Tongsheng, Z., China

Tõnsuaadu, K., Estonia

Toplan, H. Ö., Turkey

Toplan, N., Turkey

Torabi, M., USA

Torra, V., Spain

Torres, F. G., Peru

Trache, D., Algeria

Trevisan, M. G., Brazil

Trif, L., Hungary

Trikkel, A., Estonia

Trník, A., Slovakia

Trobajo, C., Spain

Tsai, K.-C., Taiwan

Tseng, J.-M., Taiwan

Tsivilis, S., Greece

Tudorachi, N., Romania

Tutunaru, B., Romania

Unluer, C., USA

Valeanu, M., Romania

Vallova, S., Czech Republic

van der Merwe, E. M., South Africa

van Ekeren, P. J., The Netherlands

Vargeese, A. A., India

Várhegyi, G., Hungary

Várhelyi Jr., Cs., Romania

Varma, I. K., India

Vasques Mendonça, A. R., Brazil

Vecchio Ciprioti, S., Italy

Vega-Gálvez, A., Chile

Venkatesu, P., India

Ventruti, G., Italy

Verma, R. K., India

Vevkatachalapathy, S., India

Vieira Rodrigues, R., Brazil

Vikulova, E., Russian Federation

Vincent, B. J., India

Vinu, R., India

Vishwakarma, P. K., India

Vladimirovich Knyazev, A., Russian Federation
Vlaev, L., Bulgaria

Vlaev, L. T., Bulgaria

Vlase, G., Romania

Vlase, T., Romania

Vogel, C., Germany

Vončina, M., Slovenia

VonRue, I., USA

Vourlias, G., Greece

Vyazovkin, S., USA

Wacławska, I., Poland

Wadeed, M. S., Egypt

Wan, Z., China

Wang, B.-I., China

Wang, C., China

Wang, C.' an, China

Wang, D.-Y., Spain

Wang, F., China

Wang, H., China

Wang, J., China

Wang, J. W., China

Wang, K., China

Wang, L., China

Wang, M., China

Wang, N., China

Wang, P., China

Wang, Q., China

Wang, Q., USA

Wang, S., China

Wang, S. B., China

Wang, W., China

Wang, X., China

Wang, X.-D., China

Wang, X. J., China

Wang, Y., China

Wang, Y.-W., Taiwan

Wang, Z., China

Wei, Q., China

Wei, Z., China

Weibing, Z., China

Weichold, O., Germany

Wesolowski, M., Poland

White, R., USA

Wiecinska, P., Poland

Wilińska, I., Poland

Wilkie, C. A., USA

Winnefeld, F., Switzerland

Worzakowska, M., Poland

Wszelaka-Rylik, M., Poland

Wu, D., China

Wu, G., China

Wu, J., USA

Wu, K., China

Wu, R.-g., China 
Wu, S., China

Wu, S.-H., Taiwan

Wyrzykowski, D., Poland

Xia, H., China

Xiao, L., China

Xiao, X., China

Xiao, Z., China

Xie, H., China

Xin, H., China

Xing, M., China

Xing, X., China

$\mathrm{Xu}, \mathrm{K}$., China

$\mathrm{Xu}, \mathrm{Q}$., China

$\mathrm{Xu}, \mathrm{R}$. , China

$\mathrm{Xu}$, S., China

$\mathrm{Xu}, \mathrm{T}$. , China

Xu, Y., China

Yamada, S., Japan

Yan, P., China

Yan, Q.-L., China

Yan, Q.-L., Czech Republic

Yan, Y., China

Yang, G., China

Yang, L., China

Yang, R., China

Yapıc1, A. N., Turkey

Yariv, S., Israel

Yarmand, H., Malaysia

Ye, L., China

Yi, L., China

Yildiz, K., Turkey

Yilmaz, V. T., Turkey

Yin, S., China

Yinmin, Z., China

You, M.-L., Taiwan

Young, G., USA

Yörük, C. R., Estonia

$\mathrm{Yu}, \mathrm{H}$. , China

Yu, J., USA

Yu, Q., China

Yuan, Y., China

Yun, L., China

Zabihi, O., Iran

Zaharescu, M., Poland

Zaharescu, T., Romania

Zaldivar, M. P., Brazil
Zalga, A., Lithuania

Zamali, H., Tunisia

Zari, N., Morocco

Zarzyka, I., Poland

Zdravkovic, J., Serbia

Zelko, R., Hungary

Zeman, S., Czech Republic

Zemanova, M., Slovakia

Zemenová, P., Czech Republic

Zeng, J.-L., China

Zhang, F., China

Zhang, G., China

Zhang, G.-Z., China

Zhang, H., China

Zhang, J., China

Zhang, J.-J., China

Zhang, J.-Q., China

Zhang, L., China

Zhang, T.-L., China

Zhang, Y., UK

Zhang, Y.-F., China

Zhang, Z., China

Zhang, Z., USA

Zhangfeng, Z., China

Zhao, B., China

Zhao, C.-w., China

Zhao, F.-q., China

Zhao, H., China

Zhao, Y., China

Zhao, Z.-B., China

Zheng, J. Y., China

Zheng, W., USA

Zheng, Z., China

Zhong, F., China

Zhou, S., China

Zhou, X., UK

Zhou, Z., China

Zhou, Z., USA

Zhu, W., China

Zohari, N., Iran

Zou, Z., China

Zulumyan, N. H., Armenia

Żyła, G., Poland

Zvereva, I. A., Russian Federation 